\title{
Effect of Pterygium on Tear Film
}

\author{
Drvmvrvprasadarao M.S. \\ Professor Of Ophthalmology, Govt. Siddartha Medical College, Vijayawada, A.P.
}

\begin{abstract}
Purpose: To investigate changes of dry eye, test results in patients who underwent pterygium surgery. Methods. Eighty patients who underwent primary pterygium surgery were enrolled in this study, from march 2014 toMarch 2015. At the baseline, 3-, 12-, and 18-month visits, measurements of BUT, and Schirmer test were performed. The patients were divided into 2 groups: Group 1, which consisted of patients in whom pterygium did not recur, and Group 2, which consisted of patients in whom pterygium recurred after surgery. Results: The prevalence rates of dry eye syndrome (DES) were lower than that at baseline and 18 months after surgery in Group 1 . In Group 2, the incidence of DES was lower after 3 months than at baseline but was similar to the baseline rate after 12 and 18 months. Conclusions: Anormal tear film function associated with pterygium. Pterygium excision improved tear film function. However, tear film deteriorated again with the recurrence of pterygium.

Keywords: Pterygium, But Test, Schirmer Test,Dry Eye,Uv Rays.
\end{abstract}

\section{Introduction}

Pterygium is a common disease of the ocular surface characterized by the invasion of fibrovascular tissue from the bulbar conjunctiva onto the cornea. It can cause chronic ocular irritation, induced astigmatism, tear film disturbances, and decreased vision secondary to growth over the visual axis. Although the exact etiology of pterygium is unknown, exposure to ultraviolet (UV) radiation is thought to be the major environmental risk factor. Age, hereditary factors, sunlight, chronic inflammation, micro trauma, and dry eye are other possible contributing factors. The most commonly accepted treatment for pterygium is surgical excision. However, the rate of recurrence after surgery is high. Several studies have used tear function tests, such as the Schirmer test or tear breakup time (BUT), to investigate the relationship between pterygium and dry eye syndrome (DES), with conflicting results. In addition, a very few studies have evaluated the effects of the excision of pterygium on tear function.

Various methods (i.e., the BUT, Schirmer, and mucus fern tests) are available for the investigation of DES. However, these tests are not always reliable, and none of them alone is sufficient for diagnosis. Therefore, in this study we aimed to investigate the changes in tear film, breakup time (BUT), and Schirmer test results in patients who had undergone pterygium surgery and to evaluate how these parameters changed when pterygium recurred after primary surgery.

\section{Materials And Methods}

Eighty eyes of 80 patients that underwent primary pterygium surgery were enrolled consecutively in this prospective study. Clinical visits were made at baseline (before surgery), 2, 7, and 20 days, and 3, 12, and 18 months after surgery. At the baseline, 3-, 12-, and 18-month visits, measurements of tear and BUT and the Schirmer test were performed by the same investigator for each patient. The presence of fibrovascular tissue with a horizontal length from limbus to cornea of $\geq 2 \mathrm{~mm}$ (measured by slit lamp biomicroscopy) was accepted as pterygium and treated by pterygium surgery. Extent of its invasion onto the cornea was assessed for determining severity of pterygium. Fibrovascular growth onto the cornea of $>0.5 \mathrm{~mm}$ recorded during the postoperative follow-up period was accepted as recurrence of pterygium. The patients were divided into 2 groups: Group 1, which consisted of patients in whom pterygium did not recur, and Group 2, which consisted of patients in whom pterygium recurred after surgery. All patients were informed about the study procedure and gave written informed consent to participate

Each patient underwent a standard ophthalmological examination to exclude patients with ocular or extra ocular diseases other than pterygium that could affect tear film function, such as blepharitis, ocular allergy, thyroid diseases, lacrimal system disorders, diabetes, collagen diseases, and use of any topical or systemic drug during the 3-month period before the examination.

Surgical Procedures. After topical and subconjunctival administration of $2 \%$ xylocaine for anesthesia, the head of the pterygium was separated and dissected away from the cornea. The pterygium was resected, the episcleral and Tenon's tissues were dissected away from the overlying sclera, conjunctival autograftwith out sutere done. 


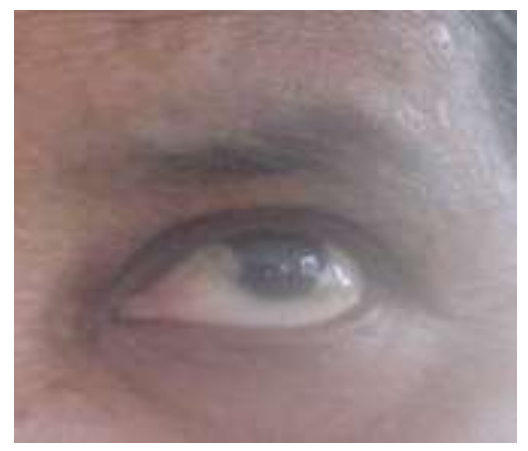

Pre operative

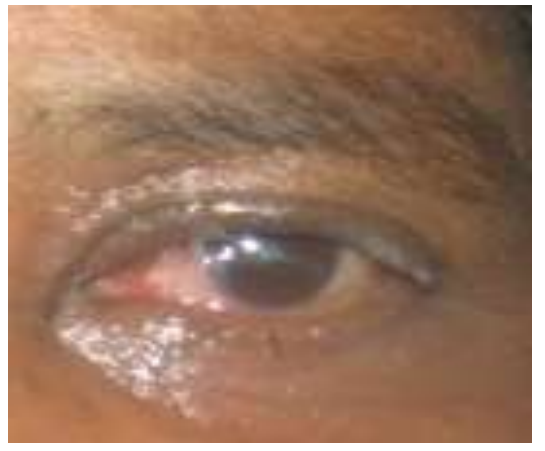

post operative

Tear Film Function Tests. The Schirmer test was performed without topical anesthesia. The length of the strip that was wet after 5 minutes was measured and accepted as the test result. The BUT was measured using fluorescein and a slit lamp with cobalt blue illumination. The average value of 2 consecutive measurements was used for analysis. The BUT was evaluated at least 30 minutes after the Schirmer test.

Table 1: The comparisons of the break-up time (BUT) and schirmer test results within the groups during the follow-up period (mean $\pm \mathrm{SD})$

\begin{tabular}{|c|c|c|}
\hline & $\begin{array}{c}\text { Group 1 } \\
(\mathrm{n}=66)\end{array}$ & $\begin{array}{c}\text { Group 2 } \\
(\mathrm{n}=14)\end{array}$ \\
\hline BUT (second) & & $10.1 \pm 3.7$ \\
\hline Baseline & $10.3 \pm 3.0$ & $10.7 \pm 3.9$ \\
\hline $3^{\text {rd }}$ month & $11.8 \pm 3.8$ & $11.2 \pm 3.7$ \\
\hline $12^{\text {th }}$ month & $11.9 \pm 3.4$ & $11.1 \pm 3.9$ \\
\hline $18^{\text {th }}$ month & $11.8 \pm 3.5$ & $12.0 \pm 3.9$ \\
\hline Schirmer test (mm) & & $12.2 \pm 3.1$ \\
\hline Baseline & $12.5 \pm 3.6$ & $12.5 \pm 3.6$ \\
\hline $3^{\text {rd }}$ month & $12.5 \pm 3.5$ & $12.7 \pm 3.6$ \\
\hline $12^{\text {th }}$ month & $12.7 \pm 3.9$ & \\
\hline $18^{\text {th }}$ month & $12.9 \pm 3.6$ & \\
\hline
\end{tabular}

Group 1 includes patients with no recurrence of pterygium after primary pterygium surgery Group 2 includes patients with recurrence of pterygium after primary pterygium surgery

\section{Statistical Analysis}

Statistical analyses were performed. All variables were distributed normally and expressed as the mean \pm standard deviation. Categorical variables were compared between the groups using the chi-square test. The Friedman test and paired -test were used for comparisons within each group. The McNemar test was used to compare prevalence rates within each group. The level of statistical significance was set at $\mathrm{P}<0.05$.

\section{Results}

There were 66 patients ( 38 male and 28 female) in Group 1 and 0 patients (10 male and 4 female) in Group 2. All recurrences of pterygium occurred between the 3rd and 18th postoperative months. The mean age was (range, 31 to 59) years in Group 1 and (range, 33 to 58) years in Group 2. The patients' age and sex ratio did not differ significantly between the groups.

The comparisons of BUT, and Schirmer test results between the groups during the follow-up period are shown in Table 1 . The BUT results changed significantly over the follow-up period within Group 1. The patients had significantly higher BUT values 3, 12, and 18 months after surgery than at baseline. However, the results of the BUT test did not change significantly within Group 2. In addition, the results of the Schirmer test did not change significantly within either group.Preoperatively, the length of the fibrovascular tissue correlated with the BUT. However, the length of the fibrovascular tissue did not correlate with the result of the Schirmer test.There was no correlation between the length of the recurrent fibrovascular tissue and the results of the dry eye tests 18 months after surgery in the recurrent pterygium group.

\section{Discussion}

This study has demonstrated that BUT values improved significantly after primary pterygium excision in Group 1. On the other hand, although the incidence of DES significantly decreased after excision of pterygium in both groups and increased again only in cases of recurrent pterygium. The BUT values of Group 2 and Schirmer test results of both groups were similar to baseline levels throughout the follow-up period. 
The preoccular tear film layer is the eye's first line of defense against environmental insults such as dryness and UV exposure. Therefore, some authors have thought that impairment of tear function could be a risk factor for diseases caused by UV exposure, including pterygium. Conversely, the reverse mechanism, that is, that conjunctival, corneal, or eyelid changes associated with pterygium disturb tear film function, has also been proposed.

Several studies have investigated the relationship between pterygium and changes in tear film function. Pterygium has been shown to be associated with abnormal tear film function, such as a shortened tear breakup time (BUT) or abnormal mucus fern patterns. However, conflicting results have also been reported. In 2 previous studies with follow-up periods of 1 and 2 months, the results of the BUT and mucus fern tests, but not the Schirmer test, improved significantly over their respective baseline values following pterygium excision. Conversely, another study with a 6-month follow-up period found no difference between the Schirmer and BUT test results at baseline and those obtained 1 and 6 months after surgery.

We believe that these contradictory results may have been obtained because the methods that were used to evaluate tear function were not objective and quantitative. The present study has shown that although the BUT test results improved after surgical treatment of pterygium with no recurrence, the Schirmer test results did not change. Therefore, we can speculate that the quantity of the tear film in patients with pterygium is adequate but that its quality or composition is abnormalAnalysis found that the recurrence rate after pterygium surgery was higher when the bare sclera technique was used than when a limbal conjunctival autograft was employed.The recurrence rate of pterygium ranges from $24 \%$ to $89 \%$ when treated with the bare sclera technique but from $1.6 \%$ to $33 \%$ when treated with conjunctival autografting. Amniotic membrane grafting has been used as an alternative to limbal conjunctival autografting, as the recurrence rate does not differ significantly between these techniques. The recurrence rate in the present study, was $9.24 \%$. We believe that the use of limbal autografting decreased the recurrence rate.

We reasonably supposed that pterygium recurrence may lead to dry eye because the pterygium disturbs tear function. Conversely, it can be speculated that dry eye may cause the recurrence of pterygium or that more severe underlying dry eye may contribute to recurrence. However, the essentially equal results for the BUT, and Schirmer test in our 2 groups prior to surgery provide strong evidence that it is pterygium recurrence that leads to dry eye. Therefore, we infer that pterygium seems to cause DES and that surgical removal of pterygium alleviates pterygium-related DES.

\section{Conflict of Interests}

None of the authors has conflict of interests with the paper.

\section{References}

[1]. J. D. Zhang, "An investigation of aetiology and heredity of pterygium. Report of 11 cases in a family," Acta Ophthalmologica, vol. 65 , no. 4, pp. 413-416, 1987.

[2]. L. Goldberg and R. David, "Pterygium and its relationship to the dry eye in the Bantu," British Journal of Ophthalmology, vol. 60, no. 10 , pp. $720-721,1976$

[3]. S. Wang, B. Jiang, and Y. Gu, "Changes of tear film function after pterygium operation," Ophthalmic Research, vol. 45, no. 4, pp. 210-215, 2011.

[4]. W. D. Mathers, "Why the eye becomes dry: a cornea and lacrimal gland feedback model," CLAO Journal, vol. 26, no. 3, pp. 159165,2000

[5]. M. A. Lemp, C. Baudouin, J. Baum et al., "The definition and classification of dry eye disease: Report of the definition and classification subcommittee of the international Dry Eye WorkShop (2007),” Ocular Surface, vol. 5, no. 2, pp. 75-92, 2007.

[6]. B. Biedner, Y. Biger, L. Rothkoff, and U. Sachs, "Pterygium and basic tear secretion," Annals of Ophthalmology, vol. 11, no. 8, pp. $1235-1236,1979$

[7]. A. Kiliç and B. Gürler, "Effect of pterygium excision by limbal conjunctival auotografting on tear function tests," Annals of Ophthalmology,

[8]. N. Di Girolamo, J. Chui, M. T. Coroneo, and D. Wakefield, "Pathogenesis of pterygia: Role of cytokines, growth factors, and matrix metalloproteinases," Progress in Retinal and Eye Research, vol. 23, no. 2, pp. 195-228, 2004.

[9]. D. Q. Li, S. B. Lee, Z. Gunja-Smith et al., "Overexpression of collagenase (MMP-1) and stromelysin (MMP-3) by pterygium head fibroblasts," Archives of Ophthalmology, vol. 119, no. 1, pp. 71-80, 2001.

[10]. G. Singh, M. R. Wilson, and C. S. Foster, "Mitomycin eye drops as treatment for pterygium," Ophthalmology, vol. 95, no. 6, pp. 813-821, 1988.

[11]. Prabhasawat P, Barton K, Burkett G, Tseng SC. Comparison of conjunctival autografts, amniotic membrane grafts, and primary closure for pterygium excision. Ophthalmology 1997;104:974-85. 